\title{
Secondary Aortoduodenal Fistula Causing Massive Upper Gastrointestinal Bleeding
}

\author{
Gonçalo Alexandrino*, David Horta and Rita Carvalho \\ Gastroenterology Department, Hospital Prof Doutor Fernando Fonseca, Portugal
}

Received: June 10, 2018; Published: June 19, 2018

*Corresponding author: Gonçalo Miguel Alves Alexandrino,Gastroenterology Department, Hospital Prof Doutor Fernando Fonseca, IC19, $2720-276$ Amadora, Portugal

\begin{abstract}
Keywords: Aortoduodenal Fistula; Endoscopy; Gastrointestinal Bleeding; Aortobifemoral; Lung Adenocarcinoma; Mild Hyperlactacidemia; Vascular Surgery; Nasogastric; Distal Duodenum; Infrarenal Aorto-Bifemoral Prosthesis; Asymptomatic; Aortal Reconstructive Surgery; Hematemesis
\end{abstract}

Abbreviations: UGE: Upper Gastrointestinal Endoscopy; CT: Computed Tomography; SADF: Secondary Aortoduodenal Fistula; ADF: Aortoduodenal Fistula; UGIB: Upper Gastrointestinal Bleeding; Aortic Aneurysm

\section{Introduction}

A 53-year-old man, with past medical history of hypertension, alcoholism, lung adenocarcinoma submitted to right upper lobectomy and peripheral artery disease with previous aortobifemoral bypass was admitted in the emergency department for acute massive hematemesis. At observation, he was hypotensive and tachycardic. A nasogastric tube was placed, with drainage of large amounts of red blood. There were no other relevant findings on physical examination. Laboratory tests revealed normocytic normochromic anemia (hemoglobin $8.8 \mathrm{~g} / \mathrm{dL}$ ) and mild hyperlactacidemia. After initial resuscitation, emergent upper gastrointestinal endoscopy
(UGE) was performed, revealing a grey regular mass covered by network-like material, adhering to a pulsatile zone of the distal duodenum wall (Figures $1 \mathrm{~A} \& 1 \mathrm{~B}$ ). The patient immediately underwent abdominal contrast-enhanced computed tomography (CT) which showed infrarenal aorto-bifemoral prosthesis, with the wall of the fourth duodenal portion not being identified, confirming the suspected diagnosis of secondary aortoduodenal fistula (SADF) (Figure 2). Vascular surgery with exclusion of the SADF and placement of endoprothesis was successfully performed. One year after the surgery, the patient remains asymptomatic.

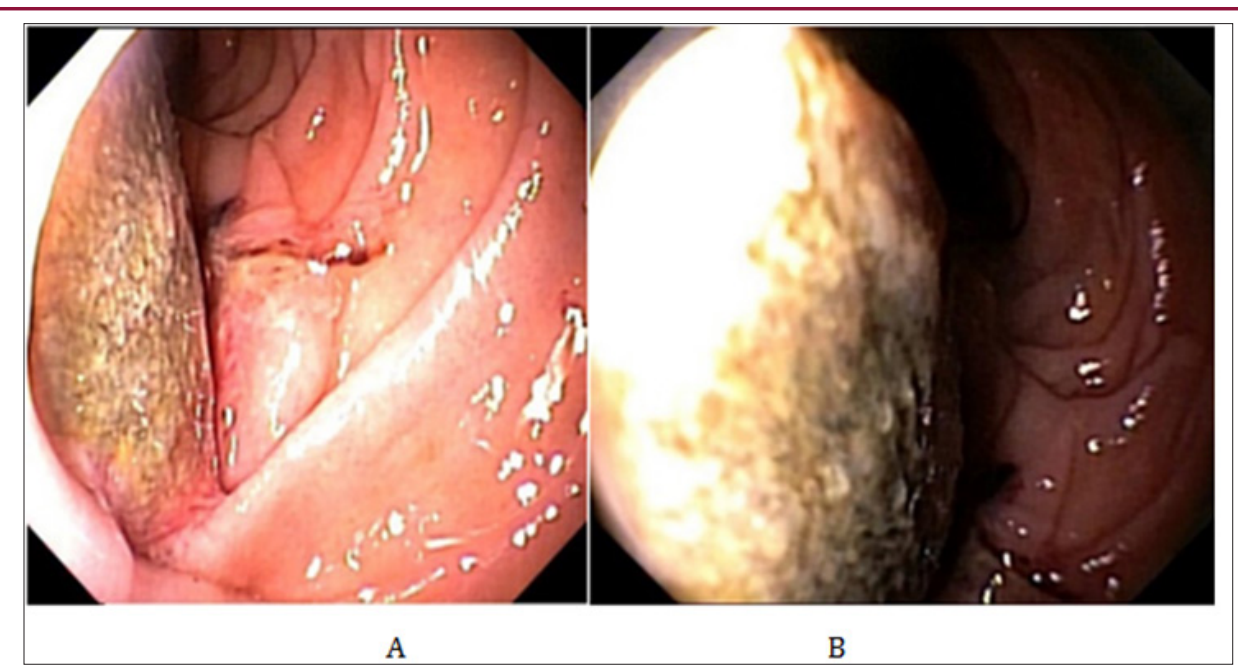

Figure 1: Upper Gastrointestinal Endoscopy: Secondary Aortoenteric Fistula of the Distal Duodenum. 


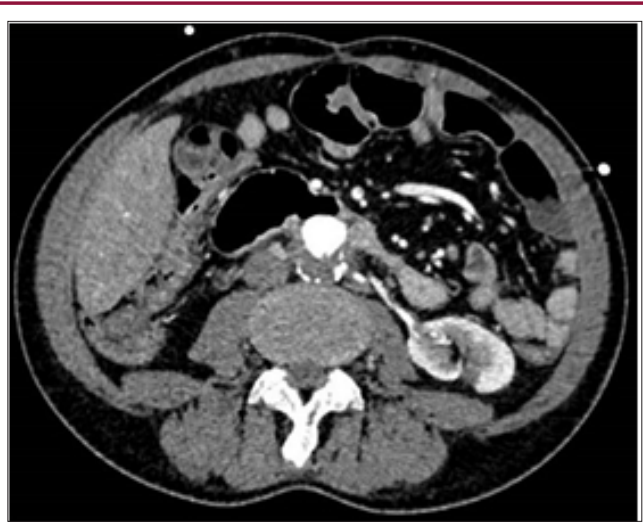

Figure 2: Abdominal Contrast-Enhanced Computed Tomography: Aortoduodenal Fistula with the Wall of the Fourth Duodenal Portion Not Being Identified.

Aortoduodenal fistula (ADF), an abnormal communication between the aorta and the bowel, is a rare cause of upper gastrointestinal bleeding (UGIB). It may be primary resulting from a spontaneous communication between an aortic aneurysm and intestinal loop, or secondary due to manipulation of aortic repair after stent/ prosthesis or graft placement. SADF is a rare (0.3-2\%) but potentially fatal complication. Non-treated ADF presenting with UGIB has a mortality rate near $100 \%$ [1]. Erosion, infection and pseudoaneu rysm are mechanisms in the pathogenesis of ADF. The most common site of SADF is in the third and fourth portion of the duodenum $(80 \%)$, like our patient. The time between the first intervention and development of fistula can range from months to years. Our patient had been submitted to aortobifemoral bypass seven years earlier. Since the clinical signs are nonspecific, it is important to have a high index of suspicion of SADF in patients that underwent aortal reconstructive surgery. The most frequent sign of ADF is UGIB. In those circumstances, the first-line diagnostic method is usually UGE, but it detects ADF in less than half of cases. In most cases, contrast CT will enable the diagnosis [2]. The treatment of choice is surgical [13]. Early postoperative mortality remains high, around 30\% [2]. In conclusion, ADF is a rare cause of UGIB, with nonspecific signs and high mortality. SADF should always be suspected in all patients who have undergone aortic graft surgery and present with gastrointestinal bleeding.

\section{References}

1. Geraci G, Pisello F, Li Volsi F, T Facella, L Platia, et al (2008) Secondary aortoduodenal fistula. World J Gastroenterol 14(3): 484-486.

2. Marolt U, Potrc S, Bergauer A, Arslani N, Papes D (2013) Aortoduodenal fistula three years after aortobifemoral bypass: case report and literature review. Acta Clin Croat 52(3): 363-368.

3. Limani K, Place B, Philippart P and Dubail D (2005) Aortoduodenal fistula following aortobifemoral bypass. Acta Chir Belg 105(2): 207-209.

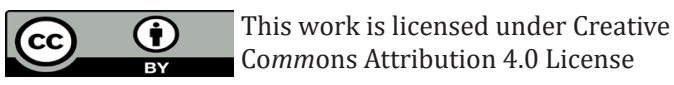

Submission Link: https://biomedres.us/submit-manuscript.php

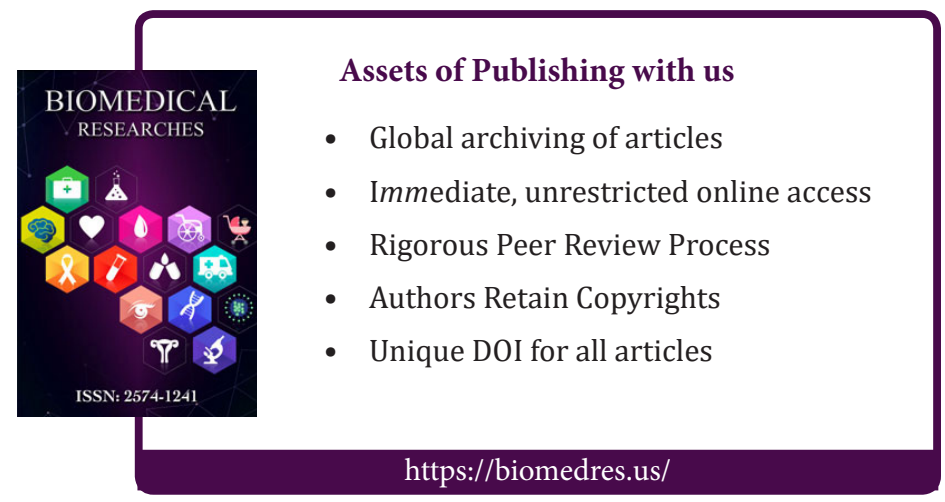

